\title{
Kinesin-8 Motors Improve Nuclear Centering by Promoting Microtubule Catastrophe
}

\author{
Matko Glunčić, ${ }^{1, \$}$ Nicola Maghelli, ${ }^{2}$ Alexander Krull, ${ }^{2}$ Vladimir Krstić, ${ }^{2,3}$ \\ Damien Ramunno-Johnson, ${ }^{2}$ Nenad Pavin, ${ }^{1,3, \dagger}$ and Iva M. Tolicic, ${ }^{2,4 *}$ \\ ${ }^{1}$ Department of Physics, Faculty of Science, University of Zagreb, 10000 Zagreb, Croatia \\ ${ }^{2}$ Max Planck Institute of Molecular Cell Biology and Genetics, 01307 Dresden, Germany \\ ${ }^{3}$ Max Planck Institute for the Physics of Complex Systems, 01187 Dresden, Germany \\ ${ }^{4}$ Division of Molecular Biology, Ruđer Bošković Institute, 10000 Zagreb, Croatia
}

(Received 5 May 2014; revised manuscript received 14 December 2014; published 18 February 2015)

In fission yeast, microtubules push against the cell edge, thereby positioning the nucleus in the cell center. Kinesin-8 motors regulate microtubule catastrophe; however, their role in nuclear positioning is not known. Here we develop a physical model that describes how kinesin- 8 motors affect nuclear centering by promoting a microtubule catastrophe. Our model predicts the improved centering of the nucleus in the presence of motors, which we confirmed experimentally in living cells. The model also predicts a characteristic time for the recentering of a displaced nucleus, which is supported by our experiments where we displaced the nucleus using optical tweezers.

DOI: 10.1103/PhysRevLett.114.078103

In interphase fission yeast cells the nucleus is found at the center of the cell [1]. This central location of the nucleus ensures symmetric cell division, because the cell divides at the site where the nucleus is located in early mitosis [2-4]. At the end of mitosis, however, the nucleus is found near the cell pole and thus must return to the cell center. In addition, between the two divisions, the nucleus must be dynamically recentered as the cell grows, because growth occurs asymmetrically with one pole growing faster than the other [5,6]. It is important for the cell to be able to move the nucleus not only for nuclear centering, but also because chromosomes move as the nucleus moves, which facilitates relocations of genomics regions [7].

The nucleus is positioned by forces exerted by microtubules (MTs) that push against the cell edge. The MTs are linked to the nucleus via MT-organizing centers (MTOCs), one of them being the spindle pole body (SPB). MTs are typically organized in 3-4 bundles that extend along the cell axis and consist of two antiparallel MTs with plus ends oriented towards the cell poles, and several short MTs in the region close to the nucleus $[1,8,9]$. MTs grow or shrink, with the switch from growth to shrinkage termed catastrophe. When the nucleus is displaced by optical tweezers [2] or centrifugation [10], MTs are able to recenter the nucleus, which is accompanied by longer lasting pushing by shorter MTs in comparison to longer MTs [10].

Centering by MT pushing has been studied theoretically by considering a dynamic array of MTs in a confined geometry [1,11-13]. In particular, centering of the nucleus in fission yeast has been explored by taking into account MT dynamics [1], and becomes more efficient if the MT catastrophe rate increases with MT length [14]. In addition, in vitro studies have shown that forces exerted by MTs interacting with a boundary may lead to centering [15-17].
PACS numbers: 87.16.Ka, 87.16.Nn, 87.16.Uv, 87.17.Aa

Dynamic properties of MTs, including catastrophe, are regulated by proteins such as kinesin- 8 motors. The effect of kinesin-8 on MT dynamics has been studied experimentally in vitro [18-24] and in vivo [20,22,25-27] as well as theoretically $[23,24,28-33]$. Fission yeast has two kinesin-8 motors, Klp5 and Klp6, which form a heterodimer [25-27,34,35]. These motors accumulate at the plus end of the MT in a MT length-dependent manner and promote catastrophe [25]. However, the role of kinesin- 8 motors in nuclear positioning is not known.

In this Letter we explore how kinesin- 8 motors affect nuclear centering. We develop a physical model that describes pushing forces exerted by MTs on the nucleus, where MT catastrophe is regulated by kinesin- 8 motors. Our model predicts improved centering in the presence of motors and a characteristic time for the recentering of a displaced nucleus, which we confirmed experimentally in living cells.

In our one-dimensional model, a cell of length $L$ has $N$ antiparallel pairs (bundles) of MTs connected to the nucleus via MTOCs (Fig. 1). The positions of the nucleus and of the $i$ th MTOC with respect to the cell center are denoted $x_{\text {nuc }}$ and $x_{i}$, respectively. The movement of the nucleus at time $t$ is described by

$$
\xi \frac{d x_{\mathrm{nuc}}}{d t}=\sum_{i=1}^{N} f_{i}
$$

Here, $\xi$ denotes the drag coefficient of the nucleus while moving through the cytoplasm and $f_{i}=\kappa \Delta x_{i}$ is the force exerted by the $i$ th MTOC. We describe the connection between the nucleus and a MTOC as a Hookean spring of stiffness $\kappa$, and extension $\Delta x_{i}=x_{i}-x_{\text {nuc }}$. We calculate the elastic force of the $i$ th MTOC as $f_{i}=f_{i}^{+}+f_{i}^{-}$, where $f_{i}^{ \pm}$ are forces exerted by MTs pushing against the right $(+)$ and left $(-)$ cell edge. MTs that are in contact with the cell 

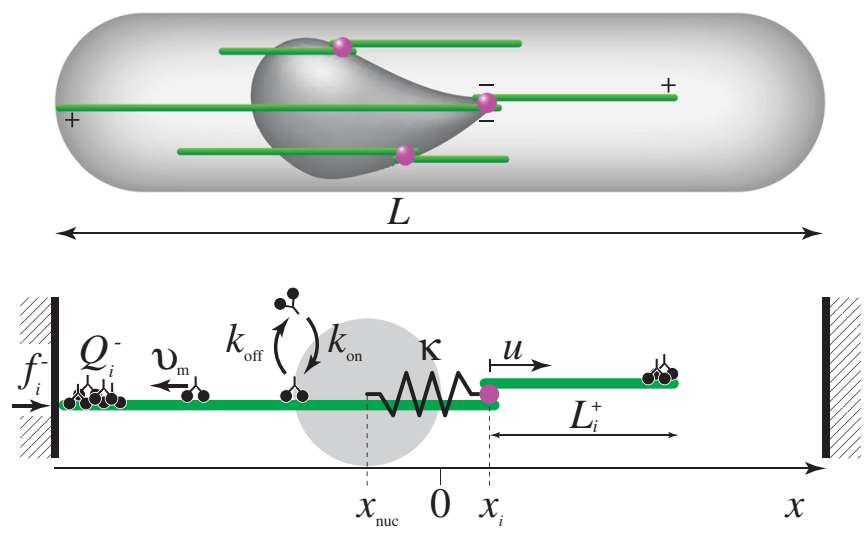

FIG. 1 (color). The model. Scheme of the cell (top) and of the model (bottom). The MTs of lengths $L_{i}^{ \pm}$(green) extend from the $i$ th MTOC (magenta) at position $x_{i}$, toward the cell periphery. MTs in contact with the cell edge exert a pushing force $f_{i}^{-}$on the MTOC. The nucleus [dark gray shape (top), gray circle (bottom)] is at position $x_{\text {nuc }}$ and is connected to the MTOC by a spring of stiffness $\kappa$. Klp5 motors (black pictograms) attach to MTs with a rate $k_{\text {on }}$, and detach with a rate $k_{\text {off }}$. Klp5 motors walk toward the MT plus ends with velocity $v_{m}$ and accumulate at the MT plus end. The number of motors at the MT plus end is denoted $Q_{i}^{-}$. The origin of the coordinate system is in the cell center. The total length of the cell is denoted $L . u$ denotes positive direction of the coordinate in the direction of the MT plus end.

edge exert a pushing force on the MTOC, which depends on the MT growth velocity [36]:

$$
f_{i}^{ \pm}=\mp f_{g, s}\left[1-\left(d L_{i}^{ \pm} / d t\right) / v_{g, s}\right]
$$

where $f_{g}$ and $f_{s}$ denote the force that stalls the MT growth and shrinkage, respectively. The length of the MTs extending from the $i$ th MTOC to the left and right is denoted $L_{i}^{-}$ and $L_{i}^{+}$, respectively. We assume that all MTs are rigid and straight; thus, those in contact with the cell edge grow and shrink with the velocity of the MTOC movement $d L_{i}^{ \pm} / d t=\mp d x_{i} / d t$ (for details on how we calculate growth and shrinkage velocity of MTs, see Supplemental Material [37]). An extension of the model, which includes elastic properties of the MTs, has a minor influence on results (Fig. S1 in Ref. [37]), and is not considered further. MTs that are not in contact with the cell edge grow with velocity $v_{g}$ and shrink with velocity $v_{s}$ in accordance with our experimental measurements (Fig. S2 in Ref. [37]).

For the MTs extending from the $i$ th MTOC, the rate of switching from growth to shrinkage, the catastrophe rate $k_{\text {cat }}$, obeys

$$
k_{\text {cat }_{i}}^{ \pm}=k_{0}+k_{1} Q_{i}^{ \pm},
$$

based on experiments from Ref. [25]. Here, $k_{0}$ denotes the catastrophe rate of MTs without motors. The parameter $k_{1}$ describes the dependence of the catastrophe rate on the number of kinesin- 8 motors at the MT's plus end $Q_{i}^{ \pm}$. Once the MT starts shrinking, it shrinks completely, which is followed by a nucleation of a new MT.

To describe kinesin- 8 motors, we use a mean field description by considering a motor density along the MT $\rho(u, t)$, which was formulated in Ref. [43], following the model introduced in Ref. [44]. For a coordinate system with the origin at the $i$ th MTOC and positive direction of the $u$ coordinate in the direction of the plus end (Fig. 1), in the low-density limit the motor density obeys [28]

$$
\frac{\partial \rho_{i}^{ \pm}}{\partial t}=-\frac{\partial\left(v_{m} \rho_{i}^{ \pm}\right)}{\partial u}+k_{\mathrm{on}} c \Theta\left(\mathrm{L}_{i}^{ \pm}-u\right) \Theta(u)-k_{\mathrm{off}} \rho_{i}^{ \pm},
$$

where the motors bind to and unbind from the MTs at rates $k_{\text {on }}$ and $k_{\text {off }}$, respectively. The concentration of motors in the cytoplasm is denoted $c$, and the velocity of motors is denoted $v_{m}$. The Heaviside step functions $\Theta\left(L_{i}^{ \pm}-u\right)$ and $\Theta(u)$ ensure that the motors bind only along the MT. We separately describe the number of motors at the plus end of the MTs extending from the $i$ th MTOC

$$
\frac{d Q_{i}^{ \pm}}{d t}=\left.\left(v_{m}-\frac{d L_{i}^{ \pm}}{d t}\right) \rho_{i}^{ \pm}\right|_{u=L_{i}^{ \pm}}-k_{\text {off }} Q_{i}^{ \pm},
$$

\begin{tabular}{|c|c|c|c|}
\hline & Parameters & Description & Source \\
\hline \multirow[t]{7}{*}{ Nuclear movement } & $L=9 \mu \mathrm{m}$ & Cell length & Measured \\
\hline & $N=3$ & Number of MT bundles & Ref. [1] \\
\hline & $f_{g}=-f_{s}=4 \mathrm{pN}$ & MT stall force & Ref. [36] \\
\hline & $v_{g}=2 \mu \mathrm{m} / \mathrm{min}$ & MT growth velocity & Measured \\
\hline & $v_{s}=6 \mu \mathrm{m} / \min$ & MT shrinkage velocity & Measured \\
\hline & $\kappa=2.5 \mathrm{pN} / \mu \mathrm{m}$ & Spring constant & Optimized \\
\hline & $\xi=1500 \mathrm{pNs} / \mu \mathrm{m}$ & Nuclear friction coefficient & Optimized \\
\hline \multirow[t]{5}{*}{ MT catastrophe } & $v_{m}=3 \mu \mathrm{m} / \mathrm{min}$ & Velocity of motors & Measured \\
\hline & With motors Without motors & & \\
\hline & $k_{0}=0.04 \mathrm{~min}^{-1}$ & MT catastrophe rate & Ref. [25] \\
\hline & $k_{\mathrm{off}}=0.25 \mathrm{~min}^{-1}$ & Motor unbinding rate & Ref. [25] \\
\hline & $K_{1}=2.1 \mu \mathrm{m}^{-1}$ & Increase of catastrophe due to motors & Ref. [25] \\
\hline
\end{tabular}

TABLE I. Parameters of the model. For measured and optimized parameters, see Ref. [37]. 
based on the experimentally observed accumulation of kinesin- 8 motors at the plus end of growing MTs (Fig. S3 in Ref. [37]).

The parameters of the model, listed in Table I, were taken from the literature, measured here, or determined as follows. Parameters $k_{0}, k_{\text {off }}$, and $K_{1}=k_{1} k_{\mathrm{on}} c / k_{\text {off }}^{2}$ were obtained by fitting the formula Eq. (S3) in Ref. [37] to the data in Ref. [25]. Note that parameter $k_{0}$ has a different value in the case with and without motors, but using a unique $k_{0}$ has a minor influence on results (Fig. S4 in Ref. [37]); parameters $\kappa$ and $\xi$ were obtained as a unique combination for which the model reproduces the standard deviations of the nucleus and the SPB measured here [Figs. 2(a)-2(c) and Fig. S5 in Ref. [37]]. Thus, the distributions of positions of the nucleus and the MTOC (SPB) calculated by the model reproduce those from the experiments [Figs. 2(b) and 2(c)]. In addition, with this choice of parameters, the results of our model are consistent with experimental data in Refs. [1], [10], [25] (see also Fig. S6 in Ref. [37]).

By solving the model in the cases with and without motors, we find that the nucleus is centered better when motors are present than when they are absent, as shown by the corresponding distributions of the nuclear position [Figs. 2(b) and 2(e)]. The MTOC is also centered better in the presence of motors, but the distribution of the MTOC position is wider than that of the nucleus, in both the case with and without motors [Figs. 2(c) and 2(f)]. We also explored how the centering of the nucleus depends on the MT number, in the cases with and without motors [Fig. S7 in Ref. [37]].

To test these theoretical predictions, we measured the movements of the nucleus and of the SPB in a $k l p 5 \Delta$ strain, which lacks the kinesin-8 motor Klp5 [Fig. 2(d)]. We observed that the nucleus is typically found farther away from the cell center in $k l p 5 \Delta$ than in wild-type cells, as shown by the 1.7-fold larger standard deviation of the distribution of the nuclear position in $k l p 5 \Delta$ cells, which is consistent with the prediction of the model [Figs. 2(b) and 2(e)]. Similarly, the division plane was positioned more asymmetrically in $k l p 5 \Delta$ cells than in wild-type cells [Fig. 2(g)]. The SPB was also typically found farther away from the cell center in $k l p 5 \Delta$ cells than in wild-type cells, and the distribution of the SPB position was wider than that of the nucleus, as predicted by the model [Figs. 2(c) and 2(f)].

In the case where a single MT touches the cell edge, our theory provides a simple formula describing how the velocity of the nucleus increases with the distance between the MTOC and the nucleus $d x_{\text {nuc }} / d t=(\kappa / \xi)\left(x_{i}-x_{\text {nuc }}\right)$ [Fig. 2(h)]. On the contrary, as the distance between the MTOC and the nucleus increases, the velocity of the MTOC decreases: $d x_{i} / d t=v_{g}\left[1-\kappa\left(x_{i}-x_{\text {nuc }}\right) / f_{0}\right]$ [Fig. 2(h)]. Note that the same formulas hold in the case without motors.

To compare the results of the model with the experiments, we measured the velocity of the nucleus and of the
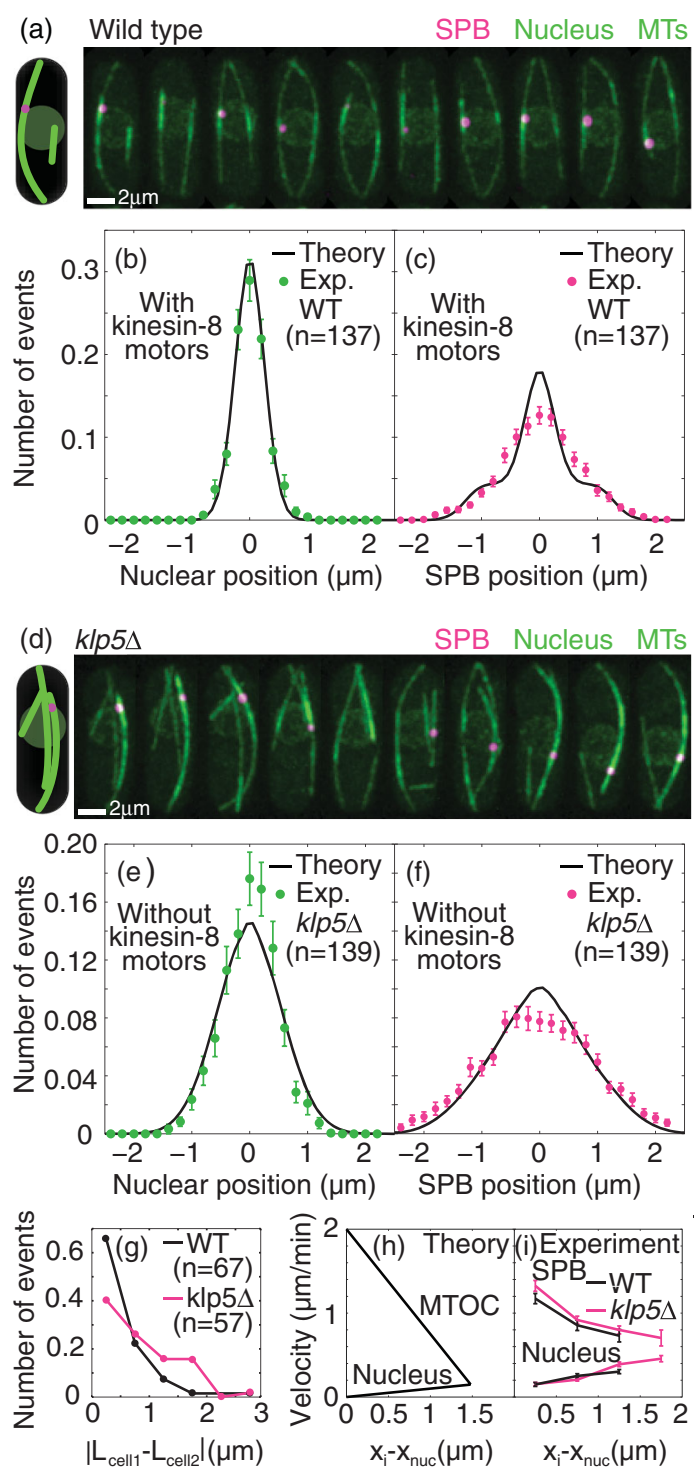

FIG. 2 (color). Centering of the nucleus and of the SPB in wildtype and $k l p 5 \Delta$ cells. Time-lapse images of wild-type (WT) (a) and of $k l p 5 \Delta$ (d) cells. Time between images is $30 \mathrm{~s}$. Schemes corresponding to the first images are shown on the left. (b), (e) Histograms of the nuclear positions, theory (black) and experiment (green). (c),(f) Histograms of the MTOC (theory, black) and of the SPB (experiment, magenta) positions. Results (b),(c) with and (e),(f) without kinesin-8 motors (for experimental procedure, see Ref. [37]). The number of events is normalized, error bars represent SEM. (g) Histograms of the position of the division plane in wild-type (black) and $k \operatorname{lp} 5 \Delta$ (magenta) cells. Fitting the histogram of the length difference of the two sibling cells, $\left|L_{\text {cell1 }}-L_{\text {cell2 }}\right|$, with a Gaussian gives a value of $0.50 \pm$ $0.02 \mu \mathrm{m}$ for wild-type cells and $1.03 \pm 0.11 \mu \mathrm{m}$ for $k l p 5 \Delta$ cells. (h) Theoretical results for the velocity of the nucleus (lower line) and of a MTOC (upper line) as a function of their distance when a single MT pushes against the cell edge. (i) Experimental results for the velocity of the nucleus (lower lines) and of the SPB (upper lines) as a function of their distance, for wild-type (black) and $k l p 5 \Delta$ (magenta) cells, when only one MT is in contact with the cell edge. The parameters for the model are given in Table I. 
(a)

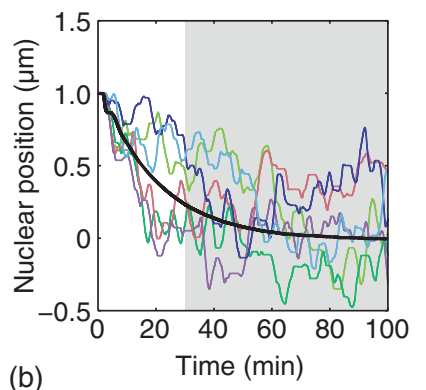

(b)

(c)
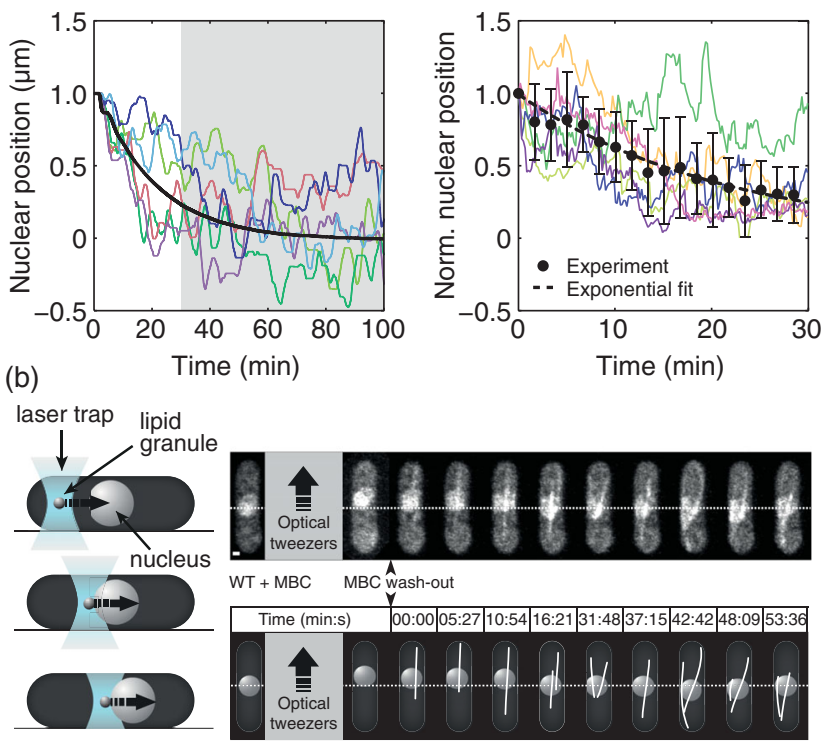

FIG. 3 (color). Recentering of a displaced nucleus. (a) Theory, the position of the nucleus as a function of time, for the starting position $1 \mu \mathrm{m}$ away from the cell center. The average curve (black) and 6 individual traces (colors) are shown. (b) Left, scheme of the optical tweezers experiment (see Ref. [37]); right, images of a cell with a displaced nucleus and the corresponding schemes. After displacing the nucleus, methyl benzimidazol-2yl-carbamate (MBC) was washed out to allow the repolymerization of MTs. (c) The experimentally measured position of the nucleus after the MBC washout as a function of time, normalized to the distance by which the nucleus was displaced. An exponential fit (dashed line) to the average data (black circles, error bars represent standard deviation) gives a characteristic time of $22 \pm 1$ min (mean \pm standard error of the mean); the colored lines represent individual wild-type cells $(n=6)$.

SPB in the cases when only one MT was interacting with the cell edge. As predicted by the model, the experimentally measured velocity of the nucleus increased and the velocity of the SPB decreased with the increasing distance between the SPB and the nucleus, in wild-type cells and in those lacking kinesin-8 motors [Fig. 2(i)]. The disagreement in the shape between the theoretical and experimental curves is most likely due to the assumption in the model that the nucleus and the MTOC are connected by a Hookean spring (a nonlinear connection is discussed in Fig. S8 of Ref. [37]).

Our model predicts that if the nucleus is displaced away from the cell center, it returns to the center obeying an exponential law with a characteristic time of $24 \mathrm{~min}$ [Fig. 3(a)], which is similar in the case without motors (Fig. S9 in Ref. [37]). To test the prediction for the characteristic time, we used optical tweezers to displace the nucleus away from the cell center in wild-type cells [2,45,46] [Fig. 3(b), see also Ref. [37]). Afterwards, we observed that the displaced nucleus returned to the cell center with a characteristic time of $22 \pm 1 \mathrm{~min}$ (mean \pm standard error of the mean, $n=6$; Fig. $3 \mathrm{c}$ ). These experimental results are consistent with the prediction from the model. The model also predicts, for the case of a displaced nucleus, a higher catastrophe rate of the MT growing towards the distal cell edge when the MT in the same bundle is in contact with the proximal cell edge in comparison with the situation of no contact (Fig. S10 in Ref. [37]). This result is in qualitative agreement with the measurements from Ref. [10].

In conclusion, we have developed a physical model that describes nuclear centering by MT pushing forces, where MT catastrophe is regulated by kinesin- 8 motors, and tested it experimentally. It has been shown in vitro that the growth velocity of a pushing MT decreases as the force on the MT increases, when the MT grows against an obstacle [36]. Our work suggests that such a force-velocity relationship holds also in vivo: when a MT was in contact with the cell edge and thus pushed on the SPB, we observed a decrease in the velocity of the SPB as it stretched the nucleus [Fig. 2(h)]. A similar conclusion could also be inferred from the angle dependence of MT growth velocities measured in vivo in Ref. [25]. Our result may be relevant for the spindle, because the MT arrangement in the spindle is analogous to the arrangement studied here turned inside out. Indeed, kinesin-8 motors help chromosome positioning on the spindle during metaphase $[26,35,47]$, but the mechanism is not known. The mechanism of centering shown here may also drive the positioning of the chromosomes on the mitotic spindle.

We thank Takashi Toda and Yvonne Caldarelli for the strains and plasmids, Britta Schroth-Diez and Jan Peychl from the Light Microscopy Facility of MPI-CBG for help with microscopy, Ivana Šaric for help with the figures, Frank Jülicher, Joe Howard, Christian Tischer, and the members of the Tolic lab for discussions. D. R.-J. was supported by a Humboldt Research Fellowship for Postdoctoral Researchers. N. P. and I. M. T. acknowledge support by the Unity through Knowledge Fund (UKF Grant No. 18/13) and the German Research Foundation (DFG). M. G. and N. M. contributed equally to this work.

*tolic@irb.hr †npavin@phy.hr *matko@phy.hr

[1] P. T. Tran, L. Marsh, V. Doye, S. Inoue, and F. Chang, J. Cell Biol. 153, 397 (2001).

[2] I. M. Tolić-Nørrelykke, L. Sacconi, C. Stringari, I. Raabe, and F. S. Pavone, Curr. Biol. 15, 1212 (2005).

[3] R. R. Daga and F. Chang, Proc. Natl. Acad. Sci. U.S.A. 102, 8228 (2005).

[4] I. M. Tolic-Norrelykke, Curr. Opin. Cell Biol. 22, 21 (2010).

[5] J. M. Mitchison and P. Nurse, J. Cell Sci. 75, 357 (1985).

[6] S. Baumgartner and I. M. Tolic-Norrelykke, Biophys. J. 96, 4336 (2009). 
[7] K. D. Kim, H. Tanizawa, O. Iwasaki, C. J. Corcoran, J. R. Capizzi, J. E. Hayden, and K. Noma, J. Cell Sci. 126, 5271 (2013).

[8] D. R. Drummond and R. A. Cross, Curr. Biol. 10, 766 (2000).

[9] J. L. Hoog, C. Schwartz, A. T. Noon, E. T. O'Toole, D. N. Mastronarde, J. R. McIntosh, and C. Antony, Dev. Cell 12, 349 (2007).

[10] R. R. Daga, A. Yonetani, and F. Chang, Curr. Biol. 16, 1544 (2006).

[11] M. Dogterom and B. Yurke, Phys. Rev. Lett. 81, 485 (1998).

[12] J. Howard, Phys. Biol. 3, 54 (2006).

[13] R. Ma, L. Laan, M. Dogterom, N. Pavin, and F. Julicher, New J. Phys. 16, 013018 (2014).

[14] D. Foethke, T. Makushok, D. Brunner, and F. Nedelec, Mol. Syst. Biol. 5, 241 (2009).

[15] T. E. Holy, M. Dogterom, B. Yurke, and S. Leibler, Proc. Natl. Acad. Sci. U.S.A. 94, 6228 (1997).

[16] C. Faivre-Moskalenko and M. Dogterom, Proc. Natl. Acad. Sci. U.S.A. 99, 16788 (2002).

[17] L. Laan, N. Pavin, J. Husson, G. Romet-Lemonne, M. van Duijn, M. Preciado López, R. D. Vale, F. Jülicher, S. L. Reck-Peterson, and M. Dogterom, Cell 148, 502 (2012).

[18] V. Varga, J. Helenius, K. Tanaka, A. A. Hyman, T. U. Tanaka, and J. Howard, Nat. Cell Biol. 8, 957 (2006).

[19] P. Grissom, T. Fielder, E. Grishchuk, D. Nicastro, R. R. West, and R. J. Mcintosh, Mol. Biol. Cell 20, 963 (2008).

[20] M. I. Mayr, S. Hummer, J. Bormann, T. Gruner, S. Adio, G. Woehlke, and T. U. Mayer, Curr. Biol. 17, 488 (2007).

[21] M. Erent, D. R. Drummond, and R. A. Cross, PLoS One 7, e30738 (2012).

[22] M. L. Gupta, Jr., P. Carvalho, D. M. Roof, and D. Pellman, Nat. Cell Biol. 8, 913 (2006).

[23] V. Varga, C. Leduc, V. Bormuth, S. Diez, and J. Howard, Cell 138, 1174 (2009).

[24] C. Leduc, K. Padberg-Gehle, V. Varga, D. Helbing, S. Diez, and J. Howard, Proc. Natl. Acad. Sci. U.S.A. 109, 6100 (2012).

[25] C. Tischer, D. Brunner, and M. Dogterom, Mol. Syst. Biol. 5, 250 (2009).

[26] R. R. West, T. Malmstrom, and J. R. McIntosh, J. Cell Sci. 115, 931 (2002).
[27] A. Unsworth, H. Masuda, S. Dhut, and T. Toda, Mol. Biol. Cell 19, 5104 (2008).

[28] C. Tischer, P. R. Ten Wolde, and M. Dogterom, Biophys. J. 99, 726 (2010).

[29] L. E. Hough, A. Schwabe, M. A. Glaser, J. R. McIntosh, and M. D. Betterton, Biophys. J. 96, 3050 (2009).

[30] A. Melbinger, L. Reese, and E. Frey, Phys. Rev. Lett. 108, 258104 (2012).

[31] D. Johann, C. Erlenkämper, and K. Kruse, Phys. Rev. Lett. 108, 258103 (2012).

[32] L. Reese, A. Melbinger, and E. Frey, Biophys. J. 101, 2190 (2011).

[33] H. S. Kuan and M. D. Betterton, Phys. Biol. 10, 036004 (2013).

[34] R. R. West, T. Malmstrom, C. Troxell, and R. J. Mcintosh, Mol. Biol. Cell 12, 3919 (2001).

[35] M. A. Garcia, N. Koonrugsa, and T. Toda, Curr. Biol. 12, 610 (2002).

[36] M. Dogterom and B. Yurke, Science 278, 856 (1997).

[37] See Supplemental Material at http://link.aps.org/ supplemental/10.1103/PhysRevLett.114.078103, which includes Refs. [38-42], for more information, methods, tables, and figures.

[38] H. Browning and D. D. Hackney, J. Biol. Chem. 280, 12299 (2005).

[39] V. Ananthanarayanan, M. Schattat, S. K. Vogel, A. Krull, N. Pavin, and I. M. Tolic-Norrelykke, Cell 153, 1526 (2013).

[40] S. Moreno, A. Klar, and P. Nurse, Methods Enzymol. 194, 795 (1991).

[41] F. Gittes, B. Mickey, J. Nettleton, and J. Howard, J. Cell Biol. 120, 923 (1993).

[42] I. Derenyi, F. Julicher, and J. Prost, Phys. Rev. Lett. 88, 238101 (2002).

[43] A. Parmeggiani, T. Franosch, and E. Frey, Phys. Rev. Lett. 90, 086601 (2003).

[44] R. Lipowsky, S. Klumpp, and T. M. Nieuwenhuizen, Phys. Rev. Lett. 87, 108101 (2001).

[45] N. Maghelli and I. M. Tolić-Nørrelykke, J. Biophoton. 1, 299 (2008).

[46] N. Maghelli and I. M. Tolić-Nørrelykke, Methods Cell Biol. 97, 173 (2010).

[47] J. Stumpff, G. von Dassow, M. Wagenbach, C. Asbury, and L. Wordeman, Dev. Cell 14, 252 (2008). 